\title{
RIPped for neuroinflammation
}

\author{
Cell Research (2017) 27:1081-1082. doi:10.1038/cr.2017.75; published online 19 May 2017
}

\begin{abstract}
Activation of the receptor interacting serine/threonine kinase (RIPK) 3 mediates an inflammatory type of cell death called necroptosis; in addition, RIPK3 has necroptosis-independent roles in inflammation, although these are not well defined. In a recent study published in Cell, Daniels and colleagues demonstrate that RIPK3 controls West Nile virus infection by promoting neuroinflammation in the central nervous system without affecting neuronal death.
\end{abstract}

The elimination of virally infected cells by programmed cell death (PCD) plays an important role in the control of viral pathogenesis. The best-known forms of PCD are apoptosis and a recently recognized form of regulated necrosis termed necroptosis. The necroptosis pathway most likely evolved as a back-up mechanism to control infection when pathogens interfere with the apoptotic machinery, and can be activated following ligation of Toll-like receptor (TLR) 3 and 4, death receptors (DR) of the tumor necrosis factor receptor family, the T cell receptor, and the cytosolic receptor DNA-dependent activator of IFN-regulatory factors (DAI). Necroptosis relies on the kinase activity of the receptor interacting serine/threonine kinase (RIPK) 3. RIPK3 is activated by RIPK1 following DR ligation, TRIF following TLR3/4 ligation, or directly by DAI. Upon activation, RIPK 3 forms amyloid structures that activate the necroptosis effector pseudokinase mixed lineage kinase-like (MLKL), which induces rupture of the plasma membrane and the release of the cellular content into the surrounding milieu [1]. By mediating necroptosis, RIPK3 thus induces inflammation of the cellular microenvironment.

Recently, several groups reported cell type-specific pro-inflammatory roles for RIPK3 that are not dependent on necroptosis. In bone marrow-derived dendritic cells (BMDCs), RIPK3 is required for TLR4-induced pro-inflammatory cytokine expression and regulates nuclear translocation of the NF- $\kappa B$ subunits RelB and p50. Furthermore, RIPK3 is needed for optimal pro-IL1 $\beta$ processing and caspase- 1 and caspase- 8 activation following TLR4 ligation on BMDCs and macrophages [2].

RIPK3 is involved in the control of a variety of viral infections, such as influenza A virus (IAV), murine cytomegalovirus (MCMV), herpes simplex virus-1 (HSV-1) and Vaccinia virus. In these settings, virally induced activation of RIPK3 appears to restrict viral replication by inducing the necroptotic death of the infected cell, thereby reducing viral burden and sustaining survival of the individual.

In a recent study published in Cell, Daniels and colleagues provide evidence for the pro-inflammatory role of RIPK3 in the control of West Nile virus (WNV)-induced encephalitis, a function of RIPK 3 that does not depend on necroptosis [3]. WNV is an RNA virus that belongs to the flaviviridae family that also comprises the Zika, Yellow fever and Dengue viruses. WNV is transmitted by mosquitos and after peripheral infection travels to and replicates in lymphoid tissues before entering the central nervous system (CNS), where it resides in neurons and can cause debilitating encephalitis. Control of neuro-infiltrative WNV infections requires neuroinflammation, the infiltration of peripheral leukocytes into the $\mathrm{CNS}$ and the elimination of the infected cells by antigen-specific $T$ cells and inflammatory myeloid cells [4]. Daniels et al. show that ripk $3^{-/}$mice succumb faster to WNV infection than wild-type, $m l k l^{t_{-}}$or casp $8^{--} \mathrm{mlkl}^{-/}$animals, suggesting that the control of WNV does not require MLKL-dependent necroptosis or caspase-8-dependent apoptosis, but rather relies on a necroptosis-independent role of RIPK3.

Subcutaneous and intracranial WNV infection of wild-type, ripk $3^{--}$and $m l k l^{--}$mice, as well as infection of neuronal cell culture experiments revealed that RIPK 3 is absolutely required for the expression of chemokines such as CCL2 and CXCL10, the known attractants for neuroinfiltrating leukocytes. Concomitantly, RIPK3 was needed for the attraction to the CNS of WNV-infected animals of peripheral immune cells, including antigen-specific, CCR $2^{+}$, $\mathrm{CXCR}^{+} \mathrm{T}$ cells and $\mathrm{CD} 45^{+}$antigen-presenting cells such as macrophages and dendritic cells. This study thus shows an important necroptosis-independent role for RIPK3 in the regulation of neuroinflammation and anti-viral immunity.

In their efforts to elucidate whether RIPK3 activation is sufficient to drive chemokine expression, Daniels and colleagues developed an animal model that we expect will prove highly valuable to the field. They made use of a chimeric RIPK3 protein fused to two copies of FKBP ${ }^{\mathrm{F} 36 \mathrm{~V}}$ (RIPK3-2×FV) $[5,6] . \mathrm{FKBP}^{\mathrm{F} 36 \mathrm{~V}}$ is a modified region of the FKBP12 protein that dimerizes in response to a synthetic bivalent homologue of rapamycin called AP1. 
The addition of AP1 to RIPK3-2FV expressing cells rapidly induced RIPK3 activation, RIPK1 recruitment and necroptosis, without the need for any upstream, potentially biasing, signals. A Rosa 26 promoter and lox-stop-lox element were introduced upstream of the RIPK3-2×FV sequence, which was further tagged with a T2A-mCherry sequence, and transgenic mice harboring this construct were generated. Cre recombinase-driven excision of the stop sequence allows cell type-specific expression of the inducible RIPK $3-2 \times \mathrm{FV}$ protein and permits the study of 'clean' RIPK3 activation in vivo. Daniels et al. crossed these mice to Meox $2^{\text {Cre }}$ animals to ubiquitiously express RIPK $3-2 \times \mathrm{FV}$ and generated primary MEFs and primary cortical neuron cultures from these animals. Consistent with previous reports using RIPK3-2×FV overexpression in in vitro settings $[5,6]$, primary MEFs rapidly died upon AP1 treatment, while the addition of AP1 did not induce cell death in primary cortical neuron cultures, but induced the expression of CCL2, indicating that the activation of RIPK3 is indeed sufficient to drive chemokine expression.

This study shows a necroptosis-independent role for RIPK3 in the expression of leukocyte-attracting chemokines during neuroinflammation. It remains unclear how RIPK3 mechanistically regulates cytokine expression, but the study of Daniels et al. may hold some hints: 1) Animals harboring a mutation that inactivates the kinase activity of RIPK1 succumb to WNV infection similar to RIPK3-deficient mice and 2) the kinase activity of RIPK1 is necessary for RIPK3-mediated CCL2 and CXCL10 expression. The role for RIPK1 in the activation of the NF- $\mathrm{KB}$ pathway and induction of pro-inflammatory cytokine expression is well studied, but does not rely on its kinase activity. Various signaling routes can lead to the activation of cytokine expressioninducing transcription factors, but the effects of RIPK1 and RIPK3 on such pathways remain unexplored.

The present study provides tantaliz- ing new insights in the necroptosisindependent functions of RIPK 3 in the regulation of neuroinflammation and furthermore provides an exciting new mouse model to study the role of RIPK3 activation in vivo.

\section{Bart Tummers ${ }^{1}$, Douglas R Green ${ }^{1}$}

${ }^{1}$ Department of Immunology, St. Jude Children's Research Hospital, 262 Danny Thomas Place, Memphis, TN 38105, USA

Correspondence: Douglas R Green

E-mail: douglas.green@stjude.org

\section{References}

1 Weinlich R, Oberst A, Beere HM, et al. Nat Rev Mol Cell Biol 2017; 18:127-136.

2 Moriwaki K, Chan FK. Cell Mol Life Sci 2016; 73:2325-2334.

3 Daniels BP, Snyder AG, Olsen TM, et al. Cell 2017; 169:301-313.

4 Shives KD, Tyler KL, Beckham JD. J Neuroimmunol 2017; 308:102-111.

5 Orozco S, Yatim N, Werner MR, et al. Cell Death Differ 2014; 21:1511-1521.

6 Tait SW, Oberst A, Quarato G, et al. Cell Rep 2013; 5:878-885. 